\title{
Same-different discriminations in the pigeon
}

\author{
RICHARD PISACRETA and KEVIN WITT \\ Ferris State College, Big Rapids, Michigan
}

\begin{abstract}
Three pigeons were trained to observe five lighted keys and to peck whichever key displayed a color different from that of the other four keys. Their above-chance performances were maintained when a novel color was introduced and when they were required to peck two nonmatching keys during each trial. The results are discussed in relation to response stereotypy and the acquisition of rule-governed (conceptual) behavior.
\end{abstract}

In addition to controlling the latency, force, topography, rate, and duration of a response, reinforcers often produce response stereotypy-the tendency to repeat precise response sequences (Eckerman \& Lanson, 1969; Pisacreta, 1982b, 1982c; Schwartz, 1980, 1981, 1982; Vogel \& Annau, 1973; Wasserman, 1977). For example, Schwartz (1980) presented pigeons with two response keys and a 5 x 5 matrix of lights. At trial onset, the upper left matrix lamp was lighted. A peck to the left response key turned off the matrix lamp and lighted the one to its right. A response on the right key turned off the matrix lamp and lit the lamp immediately below it. When the lighted matrix lamp had been moved from the upper left to the lower right position, a reinforcer was presented. Four responses to each key (in any sequence) were required to produce grain. A fifth peck to either key terminated the trial without a reinforcer. Although 70 two-key sequences were possible, each bird emitted one particular sequence during most of the trials. In a subsequent study, Schwartz (1981) employed the same procedure but required variability; that is, each bird had to use a sequence different from the preceding one. The birds developed only a few sequences and received fewer than $50 \%$ of the available reinforcers. Although persistent response stereotypy cost reinforcers, it occurred frequently. As he demonstrated in his earlier papers, once stereotypy emerges, it resists change. In a related experiment, Pisacreta (1982c) required pigeons to peck six to nine keys once each, in any sequence. The birds emitted between $.0001 \%$ and $6 \%$ of the possible sequences.

Schwartz (1982) replicated his 1980 procedure, but with human subjects, who also developed stereotyped sequences. These sequences were maintained even with intermittent reinforcement ( $50 \%$ of the trials). Additionally, the subjects reported that they believed the response sequences they emitted were necessary. They could not report the rule that determined which se-

We would like to thank Lauraine Pisacreta for editing the paper. Reprints may be obtained from R. Pisacreta, Department of Psychology, Ferris State College, Big Rapids, Michigan 49307. quences would be rewarded. Schwartz suggested that, since his subjects weren't required to learn a rule, they kept repeating whatever sequence was being reinforced. Furthermore, rewards may encourage people to repeat precisely what they have done before, and therefore may discourage variability. Apparently, humans and birds tend to adopt a strategy that produces reinforcers, and to perseverate with it whether or not it is the most efficient strategy.

In Schwartz's $(1980,1981,1982)$ experiments, the same matrix lamp (the upper left one) was lighted at trial onset. In the Pisacreta (1982c) study, the birds faced six lighted keys at trial onset. Although there were several potential response sequences available, and a few came to dominate, the sequences were comparable in efficiency. Efficiency in the present case is defined as maximizing reinforcement with the minimum required response effort. However, Pisacreta (1982b) reported that birds would perseverate in stereotyped response sequences that were very inefficient. He presented pigeons with five keys lighted with color or form stimuli. Three or four of the stimuli matched. A peck to a key changed the stimulus on the key. A reinforcer was available when each key showed the same stimulus. The most efficient strategy was to peck the one or two nonmatching stimulus keys until they matched the other three or four keys. With up to 12 stimuli available, each bird produced particular stimulus matches on 60\%-100\% of the trials. For example, when presented with three blue and two red stimuli, a bird pecked each key until the key displayed green and did not peck the two red keys until they were blue. Consequently, response efficiencies were often as low as $40 \%$. In order to perseverate in a stereotyped matching sequence, the birds often had to emit $30-40$ additional pecks per trial. The birds may not have pecked only the nonmatching stimuli on each trial because such a rule was beyond their learning capability (Premack, 1978) or perhaps because they were not required to learn the rule. The present study attempted to address this issue by trying to require the birds to learn a matching rule. 


\section{METHOD}

Subjects

Three naive White Carneaux pigeons were maintained at $80 \% \pm 15 \mathrm{~g}$ of their free-feeding weights.

\section{Apparatus}

The $35 \times 35 \times 37 \mathrm{~cm}$ operant chamber was enclosed in a sound-attenuating hull. The nine-key, $37 \times 35 \mathrm{~cm}$ response panel presented the keys in a $3 \times 3$ matrix. Stimuli were rear projected on each $2.7-\mathrm{cm}$-diam key by Industrial Electronics Engineers in-line projectors (Model 1813-1820). For reference, the keys are numbered from upper left (1) across to lower right (9). The operating force of each key was approximately $0.16 \mathrm{~N}$. The center-to-center, horizontal and vertical distances between the keys were 8.1 and $6.4 \mathrm{~cm}$, respectively. A $6 \times 6 \mathrm{~cm}$ feeder aperture centered on the panel $10 \mathrm{~cm}$ above the floor provided 6-sec access to grain. During sessions, illumination was provided only by the in-line projectors. Response Keys 1,2 , and 3 (the upper three horizontal keys) were never lighted. White noise was provided. An E\&L Instruments MMD-1 computer controlled experimental events.

\section{Procedure}

The birds were magazine trained. They were shaped to peck Key 8, which presented a white field as the discriminative stimulus $\left(\mathrm{S}^{\mathrm{D}}\right)$. Each bird was permitted to produce 100 reinforcers.

Phase 1: Three-key matching. Each trial presented three illuminated keys $(4,5$, and 6$)$. Two of the randomly chosen keys presented red and the remaining key offered blue, or vice versa (two blue keys and one red key). Each of the six trial types (e.g., red-blue-red, blue-blue-red, blue-red-blue, etc.) was presented randomly five times each during each session. During each trial, a peck to one of the two matching stimuli was recorded but had no programmed consequence. A peck to the third nonmatching stimulus changed that key to the alternate color producing a stimulus match, that is, three red or three blue keys. When the three keylights matched, they began flashing once per second and Key 8 lighted with white light. A response to Key 8 produced a reinforcer, a 3-sec intertrial interval, and the next trial. White Key 8 was used so that the pecking of any matching key or stimulus was never directly reinforced. Two or three daily 30-trial sessions (except weekends) were conducted. Phase 1 lasted 100 sessions, by which time each bird was reliably performing above chance.

Phase 2: Five-key matching. The same conditions of Phase 1 were in effect, but with the addition of two additional matching keys ( 7 and 9). Each of the 30 trials presented four red keys and one blue key or four blue keys and one red key. As before, efficient matching required one peck to the only nonmatching key and then a response to white Key 8.

Phase 3: $\mathbf{S}^{\mathbf{D}}$-cued matching. At trial onset, Key 8 was lighted with the color to peck $\left(\mathrm{S}^{\mathrm{D}}\right)$ during that trial. Three pecks to Key 8, that is, fixed ratio 3 (FR3), darkened Key 8 and lighted the matching keys. For example, Key 8 alone lighted blue. Three pecks to Key 8 darkened it and lighted Keys 4, 5, 6, 7, and 9 (four red and one blue). A peck to the blue key produced a match indicated by five flashing red keys and Key 8 reilluminated with white. A peck to Key 8 yielded a reinforcer. This phase lasted 20 sessions.

Phase 4. Over five sessions, trials were initiated with a white Key 8 . Three pecks produced the matching keys.

Phase 5: $S^{\Delta}$-cued matching. At trial onset, Key 8 displayed the stimulus color the bird should not peck $\left(S^{\Delta}\right)$ during that trial. For example, if the trial consisted of four red keys and a blue key, Key 8 presented red at trial onset. As before, three pecks to Key 8 darkened it and lighted the five matching keys. Phases 3,4 , and 5 attempted to improve the matching efficiencies established during Phases 1 and 2. Phase 5 lasted 10 sessions.
Phase 6: $S^{\triangle}$-cued matching with novel stimuli. For the next 20 sessions, green was introduced as a matching stimulus. Trials consisted of red-green, red-blue, blue-red, or blue-green stimuli. As before, Key 8 initially showed the color to avoid.

Phase 7. Over 15 sessions, the conditions of Phase 4 were replicated by initiating trials with a white Key 8 .

Phase 8. The 10 sessions of this phase reinstated the conditions of Phase 2: five-key matching without an initial response requirement to Key 8.

Phase 9: Two active keys. During the last 25 sessions of the experiment, the matching keys presented one color on three keys and a second color on the other two keys (e.g., three red and two blue, three green and two red, etc.). The birds' task was to peck each of the two keys until the color on them matched the other three keys.

\section{RESULTS}

Of primary interest was whether the birds would match efficiently, that is, would peck only the nonmatching keys. Figure 1 shows the percentage of trials on which each bird matched errorlessly (pecked only the nonmatching key) during Phases 1 and 2 . The left panel (Phase 1) indicates that each bird reliably surpassed chance within 30 sessions. Subjects B1, B2, and B3 yielded matching accuracies of approximately $40 \%$, $50 \%$, and 55\%, respectively. During Phase 2, the birds achieved $50 \%, 38 \%$, and $55 \%$, respectively. Chance performance was 33\% during Phase 1 (three keys) and $20 \%$ during Phase 2 (five keys). During Phase 1, Subjects B1, B2, and B3 maintained accuracy levels of $7 \%, 17 \%$, and $22 \%$, respectively, above chance. With two extra keys in Phase 2, the birds performed at $30 \%$, $18 \%$, and $35 \%$ above chance, respectively. Subject B1 became ill and was retired after the ninth session of Phase 2.

Figure 2 shows the percentage of trials on which Subjects B2 and B3 matched errorlessly during the remaining seven phases of the experiment. The first panel shows that requiring an observing response on Key 8 (Phase 3) improved each bird's accuracy by $7 \%-20 \%$. Not providing the SD stimuli in Phase 4 produced a slight decrease in accuracies. During Phase 5, in which Key 8

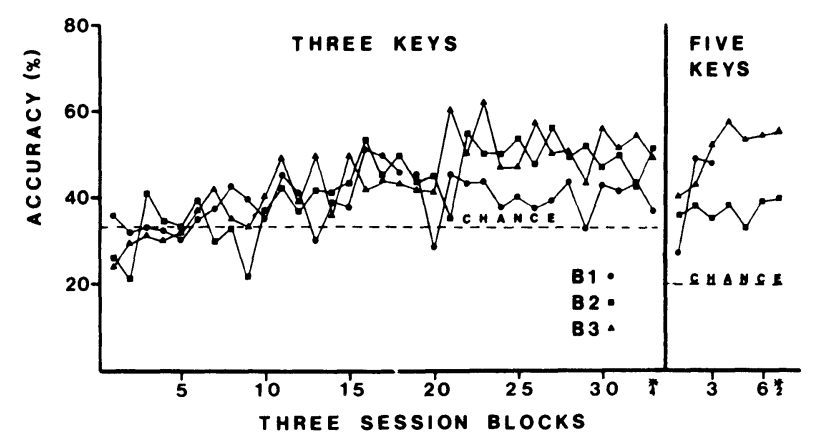

Figure 1. The percentage of the trials during Phases 1 and 2 on which each bird produced no errors, that is, pecked only the nonmatching keys. Each data point represents the mean of three sessions, except $(*)$, which represents four and two sessions during Phases 1 and 2, respectively. 


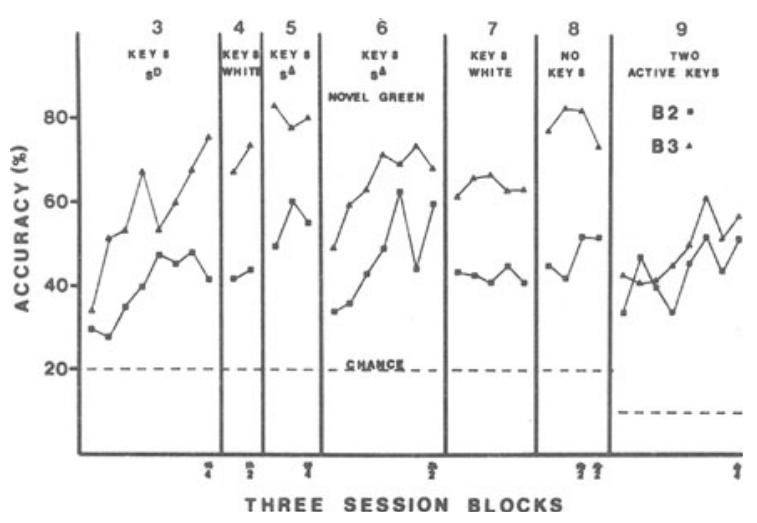

Figure 2. The percentage of the trials during Phases 3-9, inclusive, on which the birds produced no errors. The number above each panel represents the phase.

required each bird to peck the color it would have to avoid $\left(\mathrm{S}^{\Delta}\right)$, each bird produced performances superior to the performances in Phase 3. Furthermore, the accuracy levels were about $20 \%$ higher than those emitted in Phase 2. During Phase 6, in which novel green stimuli were introduced, each bird showed a slight decrease in matching levels but still performed well above chance. Not providing the $\mathrm{S}^{\triangle}$ stimuli in Phase 7 yielded 5\%-10\% matching decreases. Eliminating the observing responses to Key 8 in Phase 8 produced another increase in match-

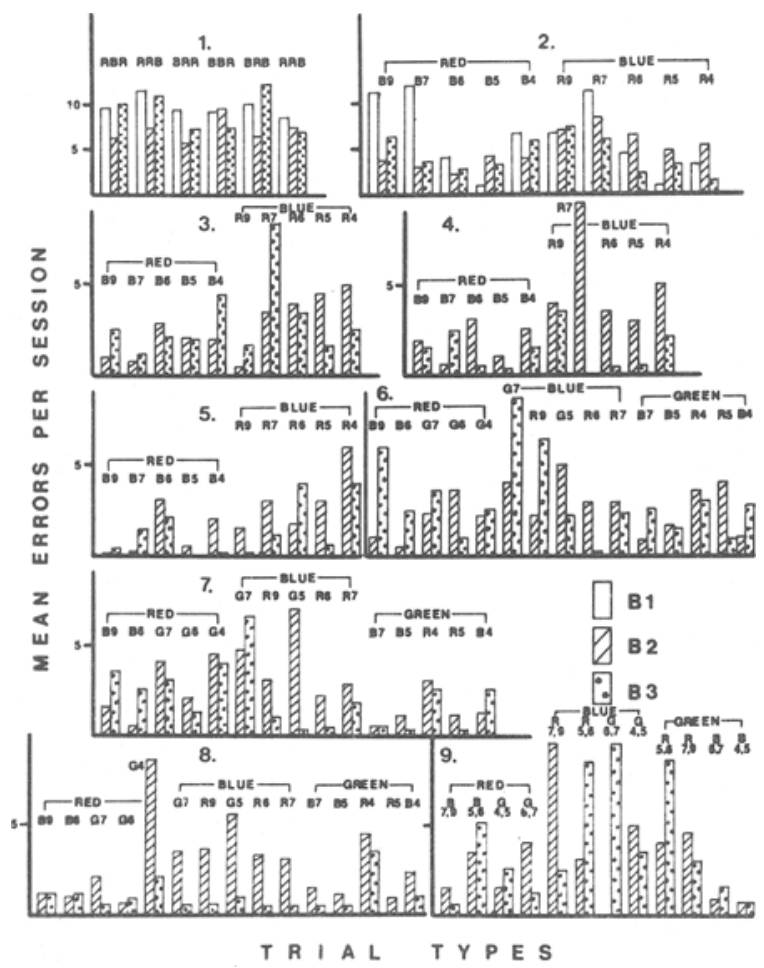

Figure 3. Mean errors per session as a function of spatial trial types. The larger number in each panel indicates the phase. The stimuli included red (R), blue (B), and green (G). See text for additional description. ing accuracies relative to the accuracies in Phase 7 . Finally, each bird performed well above chance during Phase 9 , in which two nonmatching keys had to be pecked.

Figure 3 shows the mean number of errors produced during each session as a function of stimulus spatial arrays. Panel 1, Phase 1, shows that errors were fairly equally distributed across all trial types [e.g., red-bluered (RBR), red-red-blue (RRB), etc.]. Panel 2, Phase 2, requires description. The left half of the panel shows the number of errors produced when red matches were required. For example, the first group of bars represent the mean number of errors per session during the trials that presented blue on Key 9 and red on Keys 4, 5, 6, and 7. Likewise, the other groups of bars under red represent the trials with red on all keys except 7, 6, 5, and 4 , respectively, during trial onset. Similarly, under the blue-matches group, all keys except Keys 9, 7, 6, 5, and 4 presented blue during trial onset. Panel 2 shows that Subject B1 made more errors when the correct key to peck was Key 9 and/or Key 7, relative to the other trial types. Subjects B2 and B3 exhibited similar trends. The rest of the errors are distributed fairly evenly. Panel 3, Phase 3, reveals that Subject B3 made a relatively high number of errors when a peck was required to red Key 7 in order to yield a blue match. Subject B2 produced similar results in Phase 4 . Both birds produced more errors during blue-matching trials than during red-matching trials. A comparison of Phase 5 with Phase 4 shows that the error distribution remains approximately the same, with the exception of a decrease in the number of errors made by Subject B2 when Key 7 was red and blue matching was required. During Phase 6 (novel green matches), Subject B3 made more errors on trials in which the key to peck was blue Key 9 (match red) and green Key 7 or red Key 9 (match blue) than on other trial types. Subject B2 showed a slightly uneven distribution of errors. Panel 7, Phase 7, indicates that the dominant error distributions shifted again. When observing responses were not required initially to Key 8 during Phase 8 , the dominant error distributions changed once more. Finally, during Phase 9, in which two nonmatching keys were present during each trial, the birds yielded a greater proportion of errors during blue-matching trials. Subject B3 also produced higher levels of errors during red and green matches when Keys 5 and 6 required responses. In general, the uneven error distributions suggest that the spatial stimulus arrangements influenced matching accuracy., Pisacreta (1982a) reported similar results.

\section{DISCUSSION}

Honig (1965) demonstrated that pigeons could learn a samedifferent discrimination. The birds were reinforced for pecking one of two keys if both keys showed the same stimulus and were rewarded for pecking the alternate key if both keys presented different stimuli. In a related study, Malott and Malott (1970) reinforced keypecks if both halves of a key were the same. Extinction was in effect if both halves of the key were different. 
Neither experiment required an observing response. The presence or absence of matching or identical stimuli controlled responding. Phases $1,2,4,7,8$, and 9 also required birds to make a samedifferent discrimination and peck only the nonmatching key(s), that is, oddity matching without an observing response. Phase 3, which provided the $\mathrm{S}^{\mathrm{D}}$ (color to peck) on Key 8, can be considered matching to sample with four incorrect comparison stimuli. Similarly, Phases 5 and 6 , which provided the $S^{\Delta}$ (color not to peck) on Key 8, can be considered oddity matching with four incorrect comparison stimuli. Although the birds performed reliably above chance, they did not surpass $70 \%-80 \%$ matching accuracies. Eckerman, Lanson, and Cumming (1968) reported $90 \%$ accuracies (after 55 sessions) on a matching-tosample task that did not require an initial response to the sample. The accuracy differences between their study and the present work may be due to their presenting three keys, whereas we employed five.

With conditional discriminations, birds typically do not show evidence of rule or concept learning (see Carter \& Werner, 1978, for a review). Conceptual or rule-governed behavior is defined as demonstrating a relationship between stimuli and responses that is not limited to the original training conditions. After acquiring two or three matching-to-sample problems, performance drops to near chance when a novel sample is introduced. It is often suggested that the birds learn response chains, for example, "peck red on the center key and then peck red on the side key." When transfer to a novel sample does not occur, it is implied that the matching concept is beyond the "cognitive" ability of the pigeon. However, why should the pigeon learn a matching concept if two-response chains yield reinforcement? Is it possible that reinforcing thousands of matching trials that offer one of two or three samples before introducing a novel sample may actually reduce the likelihood of transfer to the novel stimulus? Some research seems to support this view. Herrnstein and Loveland (1964) trained pigeons to peck a key only when it presented pictures containing people. The birds learned the discrimination and showed transfer with novel pictures. In a subsequent paper, Herrnstein, Loveland, and Cable (1976) demonstrated that pigeons could discriminate pictures of bodies of water, trees, and a particular person. Each session presented 80 pictures selected from a pool of $1,600-1,840$ pictures. Again, transfer to hundreds of new pictures was demonstrated. How can a pigeon fail to discriminate and match a novel color (a homogeneous field) and yet show transfer with complex photographs of water ranging from small puddles to an aerial view of the Atlantic Ocean? Similarly, Shettleworth (1983) showed that birds can recall where they stored seed several months later. Yet the delayed-matching-to-sample literature shows recall decay after only a few minutes or even a few seconds.

Blough (1979) pointed out that (1) in order to survive, animals must recognize important objects in their visual field and (2) accuracy and speed of search involves the influence of attention and information processing. Pisacreta (1983) showed that pigeons could effectively peck several different moving targets and maintain accuracy with novel targets. The present study, using 38 different problems, attempted to teach the birds a rule-peck only the nonmatching stimuli. The above-chance performances maintained with novel stimulus conditions, Phases 2, 6, and 9, suggest some degree of acquisition.

Perhaps when we are attempting to assess the "cognitive" abilities of animals, we should employ paradigms that offer several dozen problems from the onset. That is, perhaps we should present situations so complex that a rule must be acquired in order to achieve any degree of success (see Premack, 1978). These procedures should reduce what Schwartz (1982) refers to as the "negative effects of reward." That is, concept-learning tasks require variation from trial to trial. Reinforcement produces repetition, and in some cases, therefore, may actually inhibit learning. These factors could result in erroneous con- clusions about animal cognition, which would account for some of the disparity in reports addressing animal intelligence. It is possible that, to some degree, the general lack of evidence of cognitive abilities in animals may be the fault of our procedures, rather than of our subjects (see Shettleworth, 1983).

\section{REFERENCES}

BLough, D. S. Effects of the number and form of stimuli on visual search in the pigeon. Journal of Experimental Psychology: Animal Behavior Processes, 1979, 5, 211-223.

Carter, D. E., \& Werner, T. J. Complex learning and information processing by pigeons: A critical analysis. Journal of the Experimental Analysis of Behavior, 1978, 29, 565-601.

Eckerman, D. A., \& Lanson, R. N. Variability of response location for pigeons responding under continuous reinforcement, intermittent reinforcement, and extinction. Journal of the Experimental Analysis of Behavior, 1969, 12, 73-80.

Eckerman, D. A., Lanson, R. N., \& Cumming, W. W. Acquisition and maintenance of matching without a required observing response. Journal of the Experimental Analysis of Behavior, $1968,11,435-441$.

Herrnstein, R. J., \& Loveland, D. H. Complex visual concept in the pigeon. Science, 1964, 146, 549-551.

Herrnste in, R. J., Loveland, D. H., \& Cable, C. Natural concepts in pigeons. Journal of Experimental Psychology: Animal Behavior Processes, 1976, 2, 285-311.

Honig, W. K. Discrimination, generalization, and transfer on the basis of stimulus differences. In D. I. Mostofsky (Ed.), Stimulus generalization. Stanford, Calif: Stanford University Press, 1965.

Malott, R. W., \& Malott, M. K. Perception and stimulus generalization. In W. C. Stebbins (Ed.), Animal psychophysics. New York: Appleton-Century-Crofts, 1970.

Pisacreta, R. Concurrent sequential matching in the pigeon. Bulletin of the Psychonomic Society, 1982, 20, 183-186. (a)

Pisacreta, R. Preferences among stimulus matches in the pigeon. Journal of the Experimental Analysis of Behavior, 1982, 38, 191-199. (b)

Pisacreta, R. Some factors that influence the acquisition of complex, stereotyped, response sequences in pigeons. Journal of the Experimental Analysis of Behavior, 1982, 37, 359-369. (c)

Pisacreta, R. Stimulus control of the pigeon's ability to peck a moving target II: Compound stimuli and intermittent reinforcement. Psychological Record, 1983, 33, 93-116.

Premack, D. On the abstractness of human concepts: Why it would be difficult to talk to a pigeon. In S. H. Hulse, H. Fowler, \& W. K. Honig (Eds.), Cognitive processes in animal behavior. Hillsdale, N.J: Erlbaum, 1978.

Schwartz, B. Development of complex, stereotyped behavior in pigeons. Journal of the Experimental Analysis of Behavior, 1980, 33, 153-166.

Schwartz, B. Control of complex, sequential operants by systematic visual information in pigeons. Journal of Experimental Psychology: Animal Behavior Processes, 1981, 7, 31-44.

Schwartz, B. Reinforcement-induced behavioral stereotypy: How not to teach people to discover rules. Journal of Experimental Psychology: General, 1982, 111, 23-59.

Shetrleworth, S. J. Memory in food-hoarding birds. Scientific American, 1983, 248(3), 102-110.

Vogel, R., \& ANNAU, Z. An operant discrimination task allowing variability of reinforced response patterning. Journal of the Experimental Analysis of Behavior, 1973, 20, 1-6.

WASSERman, E. A. Conditioning of within-trial patterns of key pecking in pigeons. Journal of the Experimental Analysis of Behavior, 1977, 28, 213-220.

(Manuscript received for publication March 4, 1983.) 